\title{
Effect of Ag-Nanoparticles Doped in Polyvinyl Alcohol on the Structural and Optical Properties of PVA Films
}

\author{
Mahshad Ghanipour and Davoud Dorranian \\ Laser Laboratory, Plasma Physics Research Center, Science and Research Branch, Islamic Azad University, Tehran, Iran \\ Correspondence should be addressed to Davoud Dorranian; d.dorranian@gmail.com
}

Received 30 September 2013; Revised 6 December 2013; Accepted 9 December 2013

Academic Editor: Amirkianoosh Kiani

Copyright (C) 2013 M. Ghanipour and D. Dorranian. This is an open access article distributed under the Creative Commons Attribution License, which permits unrestricted use, distribution, and reproduction in any medium, provided the original work is properly cited.

\begin{abstract}
The effect of silver nanoparticles doped in PVA on the structural and optical properties of composite films is studied experimentally. Samples are PVA films of $0.14 \mathrm{~mm}$ thickness doped with different sizes and concentrations of silver nanoparticles. Structural properties are studied using X-ray diffraction and FTIR spectrum. Using the reflectance and transmittance of samples, the effect of doped nanoparticles and their concentration on optical parameters of PVA films include absorption coefficient, optical bandgap energy, complex refractive index, complex dielectric function, complex optical conductivity, and relaxation time is extracted and discussed. The dispersion of the refractive index of films in terms of the single oscillator Wemple-DiDomenico (WD) model is investigated and the dispersion parameters are calculated. Results show that by doping silver nanoparticles in PVA, number of Bragg's planes in the structure of polymer and its crystallinity are increased noticeably. Ag-O bonds are formed in the films and the bandgap energy of samples is decreased. Calculations based on WD model confirm that by doping nanoparticles, the anion strength of PVA as a dielectric medium is decreased.
\end{abstract}

\section{Introduction}

Metal nanoparticles combined polymers attracted great consideration because of the widened application goal offered by these hybrid materials [1-6]. It is well established that polymers, as dielectric materials, are excellent host matrices for encapsulation of metal nanoparticles like silver, gold, copper, and so forth, as they act both as reducing as well as capping agents and also provide environmental and chemical stability [7-9]. At the same time, these embedded nanoparticles inside the polymer matrix will also affect the properties of the host itself $[1,6,10-13]$. Particularly, polymermetal hybrid such as polymer-Ag-nanoparticles composites is promising functional materials in several fields such as optical, electrical, thermal, mechanical, and antimicrobial properties [1, 14-18]. Many reports in the literature show attempts for synthesis of metal nanoparticles based polymer nanocomposites, with the possibility of variation in their optical and electrical properties for their application in high performance capacitors, conductive inks, and other electronic components $[2,19,20]$. For their application in optoelectronic, electrical, and optical devices, biomedical science, sensors, and so forth, main key points are selection of polymer-metal nanoparticles combination, controlling the particles size, their concentration, and distribution within the polymer matrix $[2,21-23]$. Special worthy has been reached to optical properties of the nanoparticles doped in polymer film, depending on the surrounding medium [2426] and on their size, shape, and concentration [27-30]. Silver nanoparticles have received considerable attention due to their attractive physical and chemical properties [1] and it has been protected by polymers such as PVA, PVP, and PMMA. PVA could be considered as a good host material for metal due to its excellent thermostability, chemical resistance, high mechanical strength, water solubility, and moderate and dopant dependent electrical conductivity along with its consideration among the best polymers as host matrix for silver nanoparticles $[1,31]$. PVA can effectively protect the nanoparticles from aggregation $[1,9]$.

In this paper, we focus on the structural and optical property variation of the supporting polymer due to silver nanoparticles doping. Silver nanoparticles were produced 


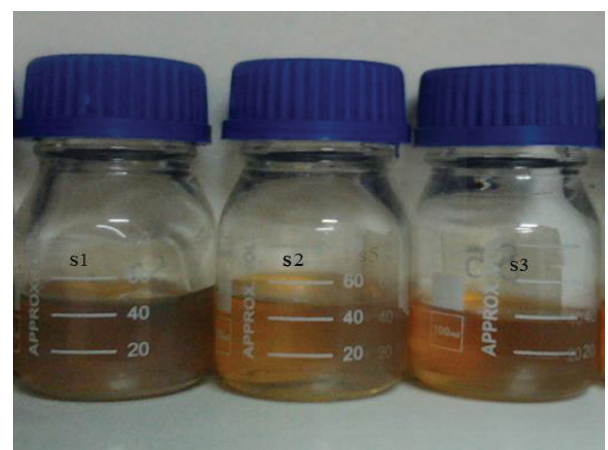

(a)

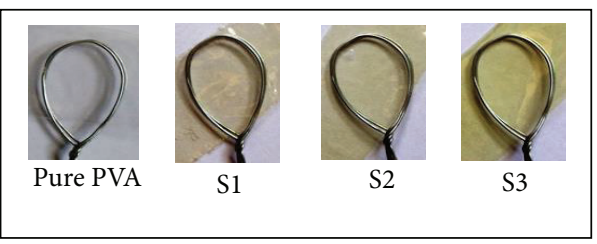

(b)

FIGURE 1: (a) Ag nanoparticle samples in distilled water and (b) pure PVA polymer film and Ag doped PVA films.

by laser ablation method. The laser ablation technique in a liquid produces proper metal nanoparticle samples to facilitate investigation of their photophysical and photochemical properties [32]. A remarkable and advantageous feature of nanoparticles prepared using this technique in contrast to those prepared using chemical synthesis is absence of uncontrolled byproducts [32, 33]. Interesting effects were observed on crystal structure and its optical properties. The noticeable main variations of optical properties of the host polymer come from surface plasmon resonance phenomena of nanoparticles dopant. The presence of silver nanoparticles embedded inside the polymer has been confirmed by the surface plasmon resonance response, which occurs at 400$420 \mathrm{~nm}$ for silver nanoparticles. The response transmittance, reflection, optical bandgap, dielectric constant, optical conductivity, dispersion refractive index, and dielectric relaxation time behavior of PVA-Ag nanoparticles films at room temperature with varying concentration of silver nanoparticles at the same thickness of silver nanoparticles doped PVA films are also investigated.

This paper is organized as follows: following the introduction in Section 1, experimental details are presented in Section 2. Section 3 is devoted to results and discussion, and Section 4 includes conclusion.

\section{Experimental Details}

Nanoparticles (NPs) were prepared by ablation of a high purity silver bulk in distilled water, using the fundamental harmonic of a Nd: YAG laser operating at $1064 \mathrm{~nm}$ with pulse width of $7 \mathrm{~ns}$ and $10 \mathrm{~Hz}$ repetition rate. Silver bulk was placed at the bottom of a water container with its surface at the focal point of a $80 \mathrm{~mm}$ convex lens. Height of water on the silver target was $12 \mathrm{~mm}$. Laser beam diameter was $2 \mathrm{~mm}$ before the lens and has been calculated to be $30 \mu \mathrm{m}$ on the surface of the target. The volume of the water in the ablation container was $20 \mathrm{~mL}$ and silver target was ablated with 500 laser pulses at different energies. Samples 1-3 were prepared with laser pulse fluencies of $1.5,2,3 \mathrm{~J} / \mathrm{cm}^{2}$, respectively.

By weighting the dried target before and after ablation process the mass of ablated Ag nanoparticles were measured to be $3.7 \times 10^{-4}, 4 \times 10^{-4}$, and $6.5 \times 10^{-4} \mathrm{~g}$ for S1, S2, and S3, respectively.

PVA films were prepared by dissolving $1 \mathrm{~g}$ of PVA powder in $20 \mathrm{~mL}$ distilled water at $57^{\circ} \mathrm{C}$. Mixture was stirred for two hours continuously to form a viscous solution. The PVA powder was provided by Merck Co., Germany. After completing desolation, $8 \mathrm{~mL}$ of silver nanoparticles suspension was added to the $20 \mathrm{~mL}$ aqueous PVA solution, and finally, samples was left to dry on a plane surface for $24 \mathrm{~h}$ at room temperature in close atmosphere to produce 3 samples of $0.14 \mathrm{~mm}$ thickness uniform silver nanoparticles doped PVA films. S1 to S3 are PVA films which are doped with samples 1 to 3 nanoparticles.

TEM micrographs were taken using CM120 system form PHILIPS Co. The X-ray diffraction (XRD) patterns of undoped and doped PVA films was measured employing STOE-XRD diffract meter with $\mathrm{Cu}-\mathrm{K} \alpha$ radiation $(\lambda=$ $1.544060 \AA)$. The Fourier transform infrared spectroscopy was done with NEXUS 870 FT-IR. The transmission and reflection spectrum of samples was recorded on a UV-VisNIR spectrophotometer from Varian Cary-500 Scan.

\section{Results and Discussion}

Nanoparticle samples and PVA doped Ag nanoparticle films are shown in Figures 1(a) and 1(b). PVA is colorless polymer and with adding Ag nanoparticles its color is changed to yellow. With increasing the concentration and decreasing the size of doped nanoparticles, color of films has become darker.

TEM images of nanoparticles are presented in Figure 2. In this set of images, the interbrain structure can be observed. Produced nanoparticles are spherical without any aggregation. The size distribution of nanoparticles can be observed in Figure 3. These graphs are plotted using the "measurement" software. We have wide range of size distribution of nanoparticles in each sample, but from samples 1 to 3 we can see that the peak of size distribution of samples is tended to smaller values. In this case the size of Ag dopants in S3 is minimum, while their concentration is maximum in comparison with S1 and S2. In contrast the size of Ag dopants in S1 is maximum, but their concentration is minimum. When the spot size and pulse width of the laser pulse are constant, increasing the laser fluence is due to increasing the laser pulse energy. If the fraction of the laser energy which is spent for ablation of nanoparticles assumed to be constant, increasing the pulse energy (photon numbers) leads to increasing the rate of ablation and increasing the pressure of the plasma plume which is formed on the surface of the target during the laser ablation process. The first phenomenon will increase the concentration of produced nanoparticles and the second phenomenon leads to decreasing the size of nanoparticles. 

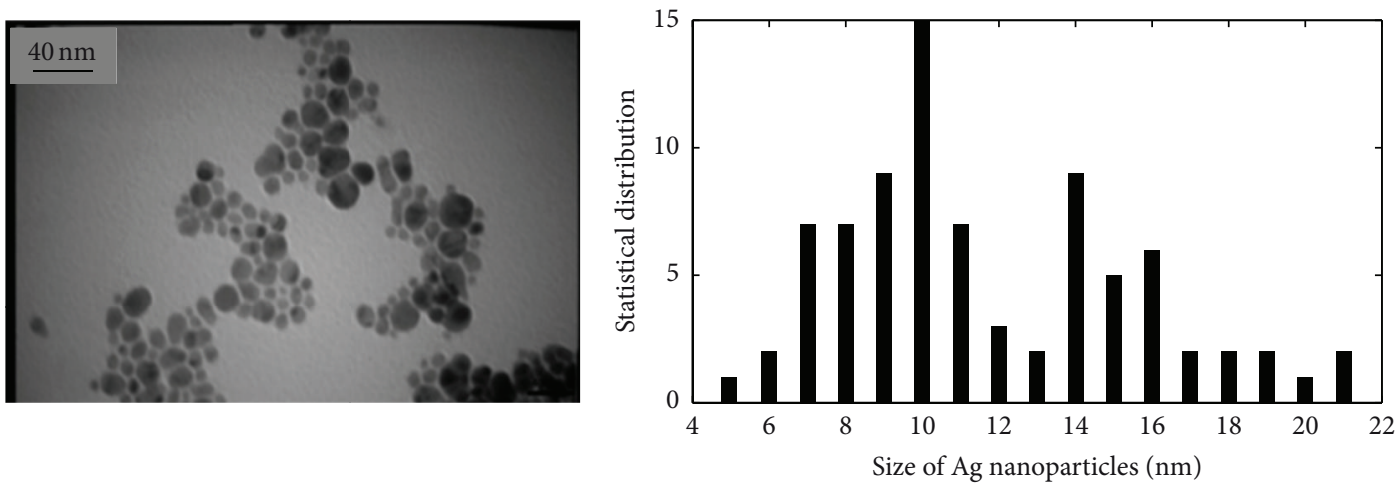

(a)
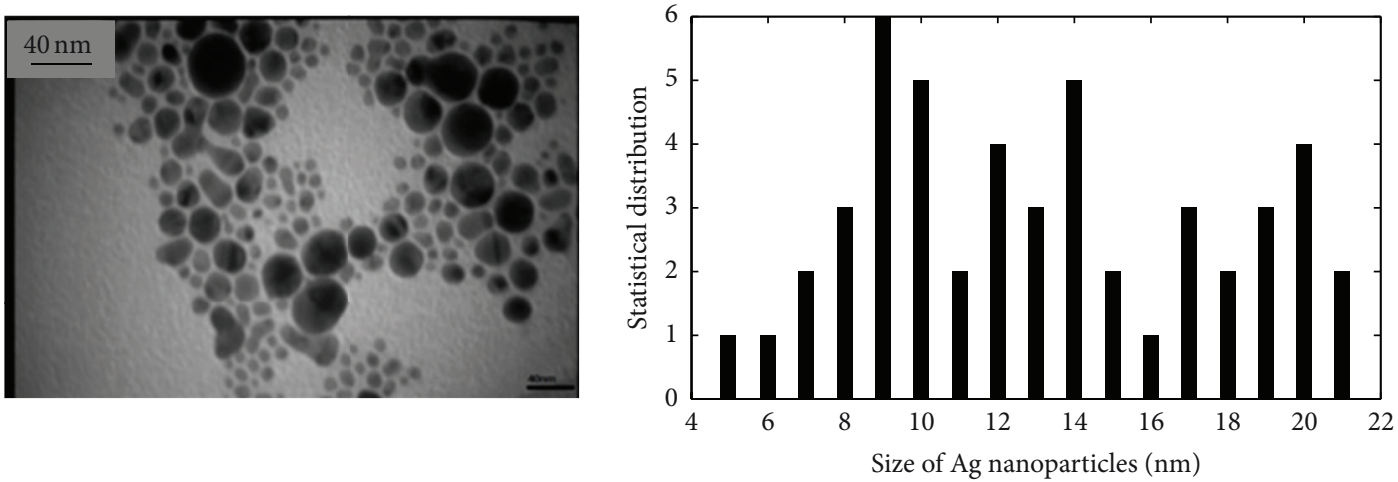

(b)
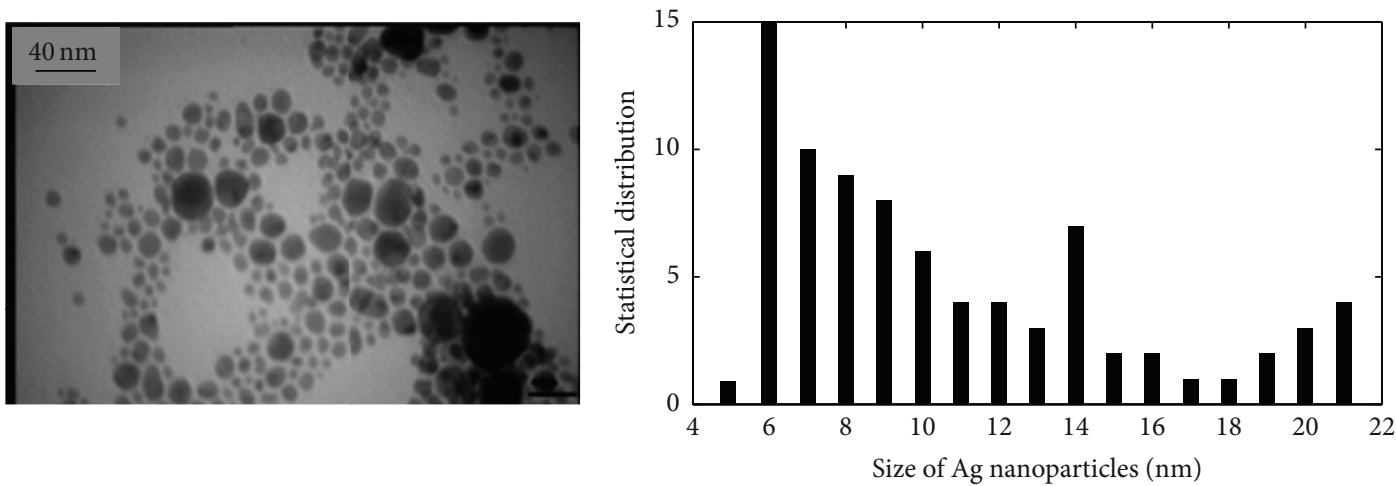

(c)

FIGURE 2: TEM image and size distribution of Ag nanoparticle generated in distilled water with laser ablation method. (a) S1, (b) S2, and (c) S3.

XRD spectrum of the Ag nanoparticles and pure PVA polymer films and PVA doped Ag nanoparticles are shown in Figures 3(a)-3(c). The diffraction pattern of undoped PVA indicates a diffraction bands at $2 \theta=13.88^{\circ}, 16.76^{\circ}$, $25.4^{\circ}, 42.12^{\circ}$, and $48.88^{\circ}$. It is well known that the peaks at $2 \theta<20^{\circ}$ are due to crystalline nature of PVA polymer molecules, which may be as a result of strong intermolecular and intramolecular hydrogen banding between the PVA chains $[7,34]$. The peaks at angles larger than $20^{\circ}$ may be due to impurities. The X-ray diffraction peaks of $\mathrm{Ag}$ nanoparticles occur at $2 \theta=38.15^{\circ}, 44.39^{\circ}, 64.74^{\circ}, 77.5^{\circ}$, and $81.6^{\circ}$ corresponding to reflections from the $\langle 111\rangle,\langle 200\rangle$, $\langle 220\rangle,\langle 311\rangle$ and $\langle 222\rangle$ planes of Ag, FCC lattice structure, respectively $[35,36]$. All peaks observed in the sample of Ag nanoparticles have also been recreated in polymer films doped with silver nanoparticles. The peaks of polymer Xray diffraction pattern at $2 \theta>20^{\circ}$ have been removed after doping. The peak at $2 \theta=42.12^{\circ}$ in pure PVA is observed to shift up by about $2.5^{\circ}$ degree in PVA doped Ag nanoparticles. This shift might be due to changes in the $d$ spacing values of the corresponding planes. The intensity of diffracted X-ray photons from films has been increased noticeably after the doping process. It may be due to two reasons. For $2 \theta<20^{\circ}$, increasing the peaks intensity is due to increasing the number 


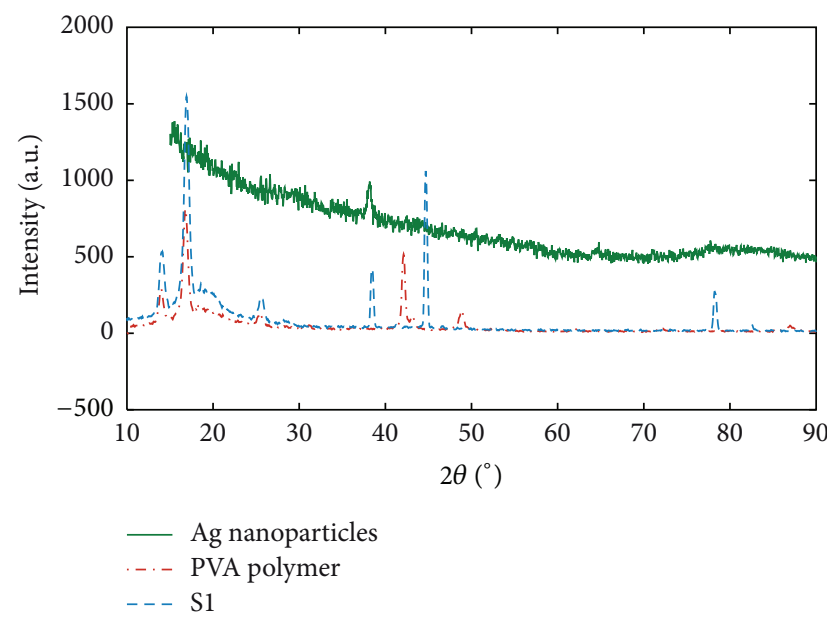

(a) $\mathrm{S} 1\left(1.5 \mathrm{~J} / \mathrm{cm}^{2}\right)$

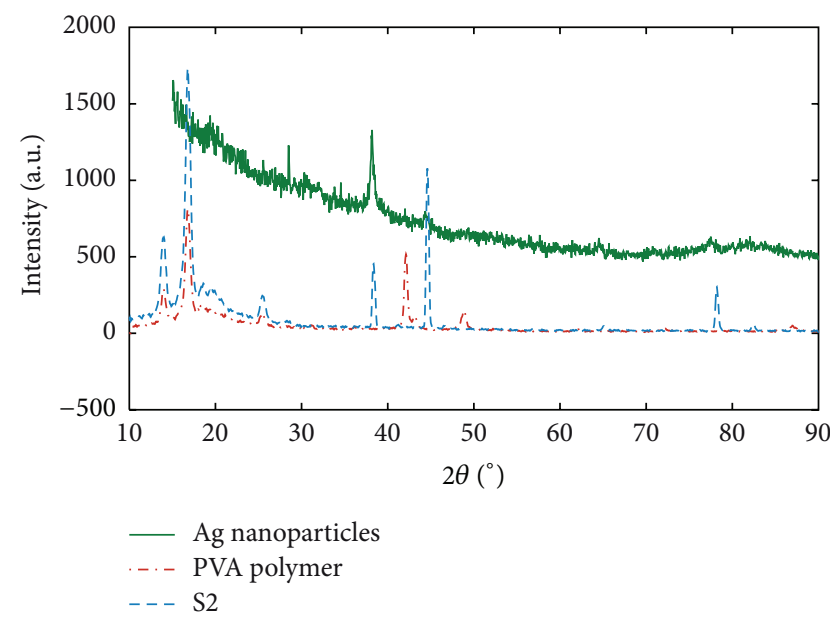

(b) $\mathrm{S} 2\left(2 \mathrm{~J} / \mathrm{cm}^{2}\right)$

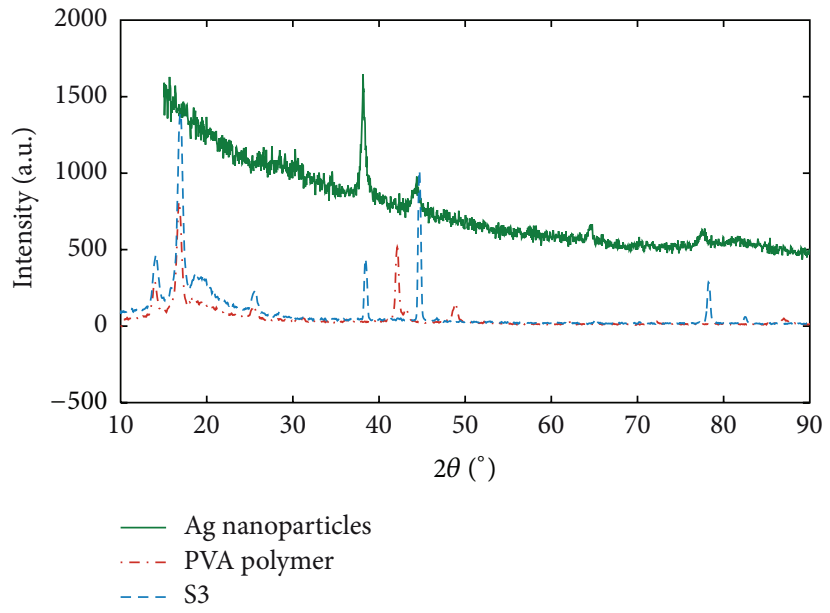

(c) $\mathrm{S} 3\left(3 \mathrm{~J} / \mathrm{cm}^{2}\right)$

FIGURE 3: X-ray diffraction patterns of Ag nanoparticles, PVA polymer, and Ag nanoparticle doped PVA films; (a) S1, (b) S2, and (c) S3.

of PVA chains after Ag doping. Decreasing the intensity of peaks in FTIR spectrum confirms that after doping, number of PVA chains are increased in the structure of the films. Same results have been observed by Mahendia et al. and Gautam and Ram [7, 34]. For $2 \theta>20^{\circ}$, increasing the intensity of XRD peaks is due to increasing the number of crystallographic planes at certain angles.

The Fourier transform infrared spectroscopy (FTIR) spectra of pure PVA and doped films are shown in Figure 4. All spectra exhibit the characteristic absorption bands of pure PVA which are $3580,2974,1740,1570,1460$, and $845 \mathrm{~cm}^{-1}$ $[37,38]$. It can be noticed that these treatments cause some observable changes in the spectral features of the samples in the range $1100-500 \mathrm{~cm}^{-1}$ (fingerprint region) apart from new absorption bands and slight changes in the intensities of some absorption bands. The new bands may be correlated likewise with defects induced by the charge transfer reaction between the polymer chain and the dopant. The vibrational peaks at 3580,2974,1740,1460, and $845 \mathrm{~cm}^{-1}$ are assigned to $\mathrm{O}-\mathrm{H}$ stretching, $\mathrm{C}-\mathrm{H}$ stretching, $\mathrm{C}=\mathrm{O}$ stretching, $\mathrm{C}-\mathrm{H}$ bend of $\mathrm{CH}_{2}$, and $\mathrm{CH}$ rocking of PVA, respectively [39, $40]$. Further, the vibrational peaks found in the range 1130$650 \mathrm{~cm}^{-1}$ may be attributed to $\mathrm{Ag}-\mathrm{O}$, which indicate that silver nanoparticles doped in the PVA polymer matrix [37]. The experimental data given in Figure 4 indicate an increase in the vibrations of $\mathrm{O}-\mathrm{H}, \mathrm{C}-\mathrm{H}$, and $\mathrm{C}=\mathrm{O}$ groups in the PVA matrix after adding Ag nanoparticles directly in the PVA film. Such changes in $\mathrm{O}-\mathrm{H}, \mathrm{C}-\mathrm{H}$, and $\mathrm{C}=\mathrm{O}$ vibrations have been observed in other report [40]. After doping Ag, some polymers chains have been broken and some other chains have been formed instead. Increasing the FTIR spectrum in the range of 1400 to $1600 \mathrm{~cm}^{-1}$ corresponds to $\mathrm{C}-\mathrm{H}$ bond of $\mathrm{CH}_{2}$ and shows the broken chains. Ag nanoparticles have generated new bonds in this range. Decreasing the FTIR spectrum in the range of 2500 to $3700 \mathrm{~cm}^{-1}$ shows the produced polymer chains corresponds to $\mathrm{O}-\mathrm{H}$ stretching and C-H stretching bonds.

The variation of transmittance $(T)$ and reflectance $(R)$ as a function of wavelength for pure PVA polymer film and samples 1 to 3 were recorded at room temperature and 


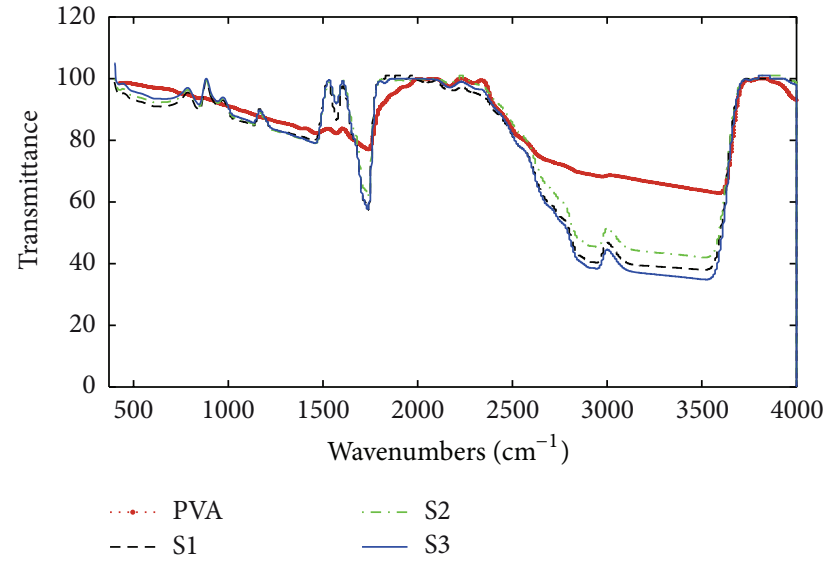

FIGURE 4: FTIR spectrum of PVA pure film and Ag nanoparticle doped in PVA polymer films.

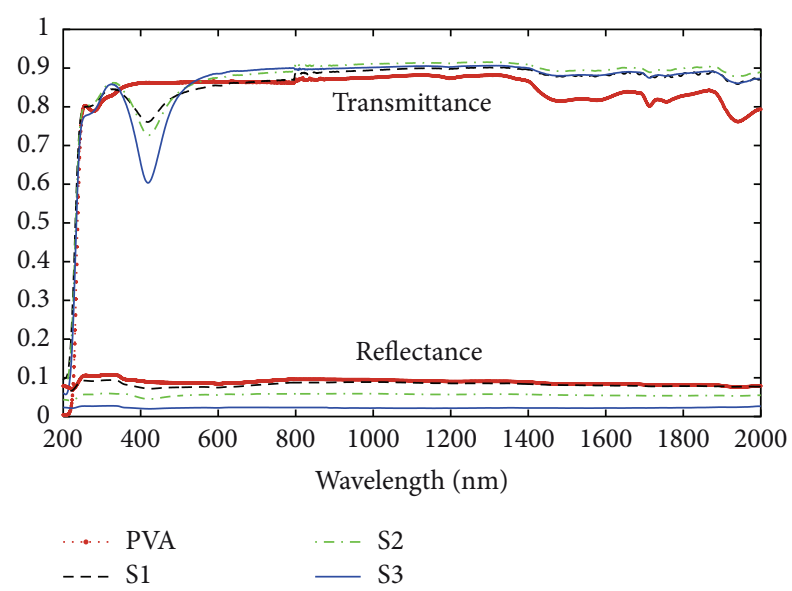

FIGURE 5: Optical transmittance and reflectance spectrum of samples.

are shown in Figure 5. Pure PVA is a colorless polymer without any noticeable absorption in the visible range. The sharp increase observed in transmittance spectrum in the range of 210 to $248 \mathrm{~nm}$ is due to the presence of the PVA polymer bandgap [1]. This figure clearly indicates that after adding nano-Ag in PVA polymer, a valley at $419 \mathrm{~nm}$ has been created, that its intensity continuously increasing with increasing concentration of the dopant. This new valley is attributed to the formation of charge transfer complexes [41]. The appearance of this valley in the visible region is due to the surface plasmon resonance (SPR) nature of the $\mathrm{Ag}$ nanoparticles embedded in PVA polymer dielectric medium.

After doping Ag nanoparticles in PVA polymer, the reflection, with increasing concentration of silver nanoparticles, due to local fluctuations charged particles, declined.

The optical absorption coefficients of samples are evaluated from the transmittance data using [42]

$$
\alpha=\frac{1}{d} \ln \left[\frac{(1-R)^{2}}{2 T}+\sqrt{\frac{(1-R)^{4}}{4 T^{2}}+R^{2}}\right],
$$

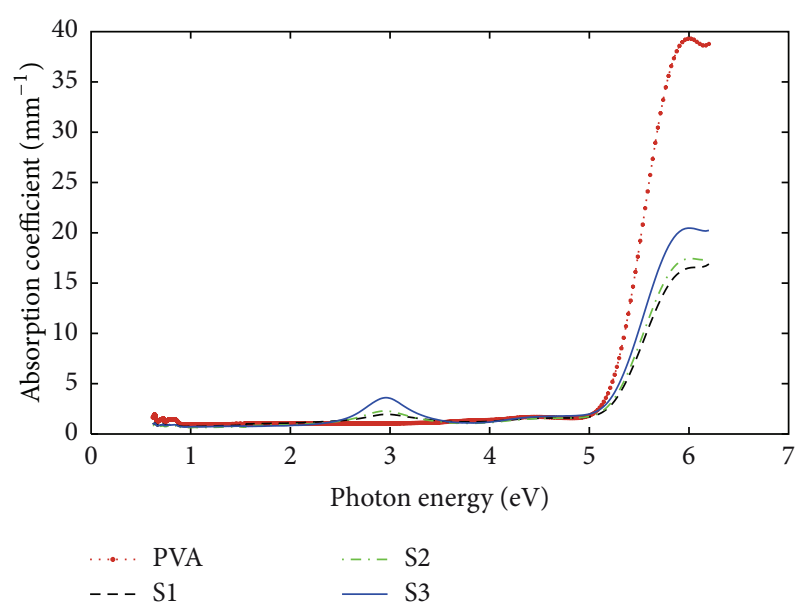

Figure 6: Optical absorption coefficient of films.

where $T$ and $R$ are the transmittance and reflection, respectively, $\alpha$ is the absorption coefficient, and $d$ is the thickness of the films. Figure 6 presents the optical absorption coefficients for undoped and nano-Ag doped PVA films versus photon energies. The absorption peak at $2.95 \mathrm{eV}$ for $\mathrm{Ag}$ nanoparticles doped in PVA polymer films represents the characteristic surface plasmon resonance dedicated to silver nanoparticles. The presence of nanoparticles in the polymer films could be conveniently followed by monitoring the plasmon absorption peaks in the absorption spectrum. The larger absorption peak appeared in UV range is due to the energy gap of the PVA polymer which decreases owing to increasing the concentration of $\mathrm{Ag}$ nanoparticles in the structure of the films. The position of the absorption edge was determined by extrapolating the linear part of $\alpha$ versus $h \nu$ curves to zero absorption value [41]. The band edge showed a decrease with increasing concentration of Ag nanoparticles in PVA matrix. The absorption edge shifts towards higher wavelength, indicating the decrease in the optical bandgap for the doped films. Shift of the absorption edge in the UV region is due to changes in the electron hole in the conduction and valence bands.

The most used method for estimation of the bandgap energy from optical measurement is the one proposed by Tauc and Grigorovici [43]. The optical bandgap energy of samples was deduced from the intercept of the extrapolated linear part of the plot of $(\alpha E)^{1 / 2}$ versus the photon energy $E$ with abscissa (Figure 7). This follows by the method of Tauc where

$$
\alpha E=B\left(E-E_{g}\right)^{p} .
$$

In this equation, $\alpha(\omega)$ is the absorption coefficient, $E$ is the photon energy, $B$ is a factor that depends on transition probability and can be assumed to be constant within the optical frequency range, and the index $p$ that is related to the distribution of the density of states is an index which assumes the values $1 / 2,3 / 2,2$, and 3 depending on the nature of electronic transition. Taking $p=2$, which corresponds to indirectly allowed transition in pure PVA film and nanoAg doped PVA films, the bandgap energies of films were 


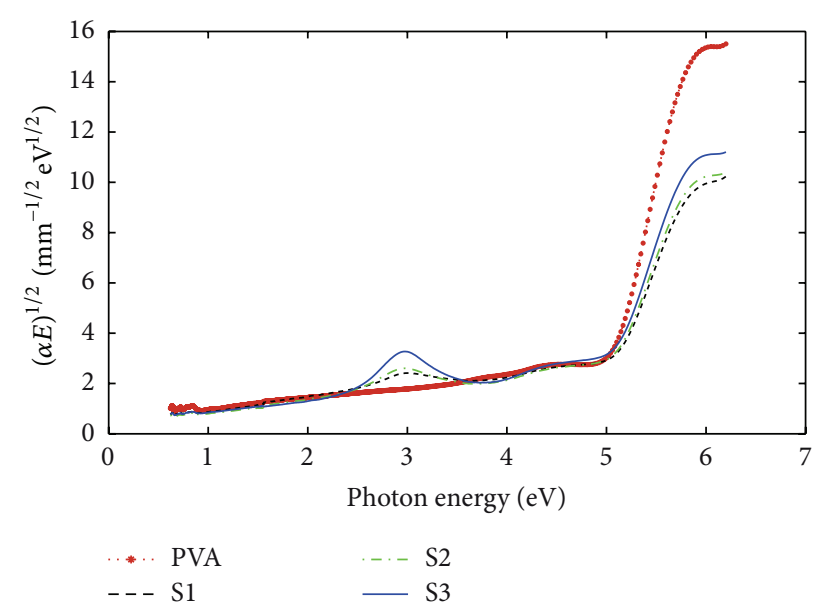

FIGURE 7: $(\alpha E)^{1 / 2}$ versus photon energy to illustrate Tauc method.

calculated. In an indirect gap, a photon cannot be emitted because the electron must pass through an intermediate state and transfer momentum to the crystal lattice. The energy gap of pure PVA sample is equal to $4.96 \mathrm{eV}$, and with increasing the concentration of the Ag-nanoparticles in the structure of films, bandgap energy is decreased. The extracted bandgap energy of sample are 4.87, 4.84, and $4.78 \mathrm{eV}$, for sample, $1-3$, respectively. The incorporation of the silver nanoparticles, irrespective of their methodology of synthesis, also affects the bandgap of the involved polymer system. This also confirms the presence of the inorganic fillers inside the host [1]. The variation of the calculated values of optical bandgap reflects the role of formation of Ag-nanoparticles in modifying the electronic structure of the PVA matrix [41, 44]. These Ag-nanoparticles may be responsible for the formation of localized electronic states in the Highest Occupied Molecular Orbital-Lowest Unoccupied Molecular Orbital (HOMO-LUMO) gap. These localized electronic states dominate the optical and electrical properties vis-à-vis their role as trapping and recombination centers, thus enhancing the low energy transitions leading to the observed change in optical bandgap. The decrease in the optical bandgap also reflects the increase in the degree of disorder in the films which arises due to the change in polymer structure $[44,45]$.

Optical properties, such as complex refractive index and dielectric constant for a certain range of wavelength between ultraviolet and near infrared, are important criteria for the selection of fabricated films for various applications. The refractive index is one of the fundamental properties of a material, because it is closely related to the electronic polarizability of ions and the local field inside the material $[8$, 9]. Thus, to further understand the interaction of Ag nanoparticles with PVA matrix, optical properties such as complex refractive index and dielectric constant have been calculated using the fundamental relations of photon transmittance $(T)$, reflectance $(R)$, and absorbance $(A)$. The complex refractive index is $n=n+i k$, that $n$ is the real part and the extinction

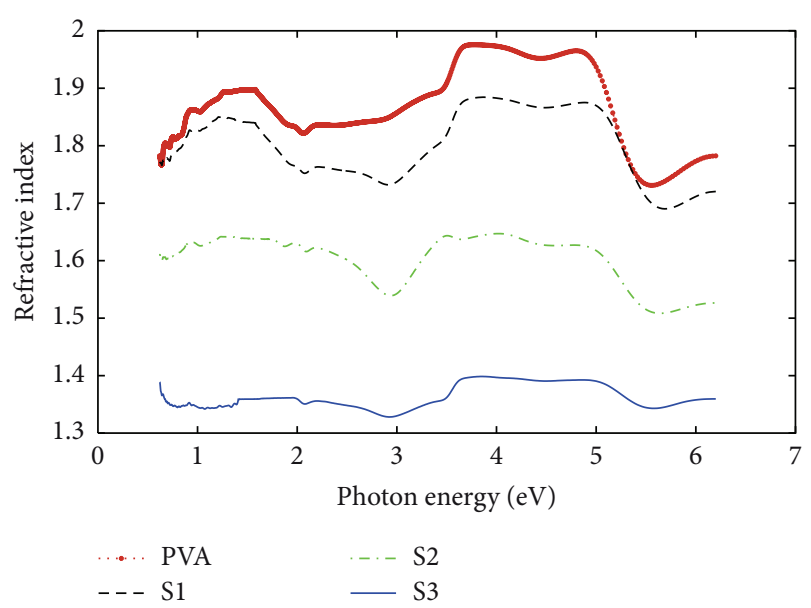

FIgURE 8: The refractive index of films.

coefficient $k$ is the imaginary part. The refractive index $n$ of the films was calculated using the following equation [46]:

$$
n=\left(\frac{1+R}{1-R}\right)+\sqrt{\frac{4 R}{(1-R)^{2}}-k^{2}},
$$

in which $k=\lambda \alpha / 4 \pi$. Figures 8 and 9 show the photon energy dependence of refractive index and the extinction coefficient for pure PVA and nano-Ag doped PVA films. It can be discerned from Figure 8 that the refractive index of nano-Ag doped PVA films is lower than the refractive index of pure PVA and it decreases with increasing concentration of Ag nanoparticles in PVA matrix. This property is inherent in all conductors and due to localized fluctuation of charged particles in medium. Also decreasing the value of refractive index may be an indication of low density of films, which leads to increasing the interatomic spacing [45]. This is due to formation of intermolecular hydrogen bonding between Agnanoparticles and the adjacent $\mathrm{OH}$ groups. The dependence of the refractive index on the film density can be discussed by the well-known Clausius-Mossotti relation [46].

The extinction coefficient $k$ describes the properties of the material with respect to light of a given wavelength and indicates the absorption changes when the electromagnetic wave propagates through the material. In Figure 9, the extinction coefficient $k$ of the doped samples have a peak at $E=$ $2.95 \mathrm{eV}$, which increases with increasing concentration of Ag nanoparticles in PVA dielectric medium. The extinction coefficient $k$ increases due to surface plasmon absorption in doped samples, while the refractive index decreases in this region. There is anomalous dispersion regions when $2.25<$ $E<2.95 \mathrm{eV}$ and $E>4.85 \mathrm{eV}$, as well as normal dispersion when $2.95<E<3.45 \mathrm{eV}$ for doped samples.

The complex dielectric function is $\varepsilon=\varepsilon_{r}+\varepsilon_{i}$, where $\varepsilon_{r}$ is the real part and $\varepsilon_{i}$ is the imaginary part of dielectric constant. The real and imaginary parts of dielectric constant are expressed as

$$
\begin{gathered}
\varepsilon_{r}=n^{2}-k^{2}, \\
\varepsilon_{i}=2 n k .
\end{gathered}
$$




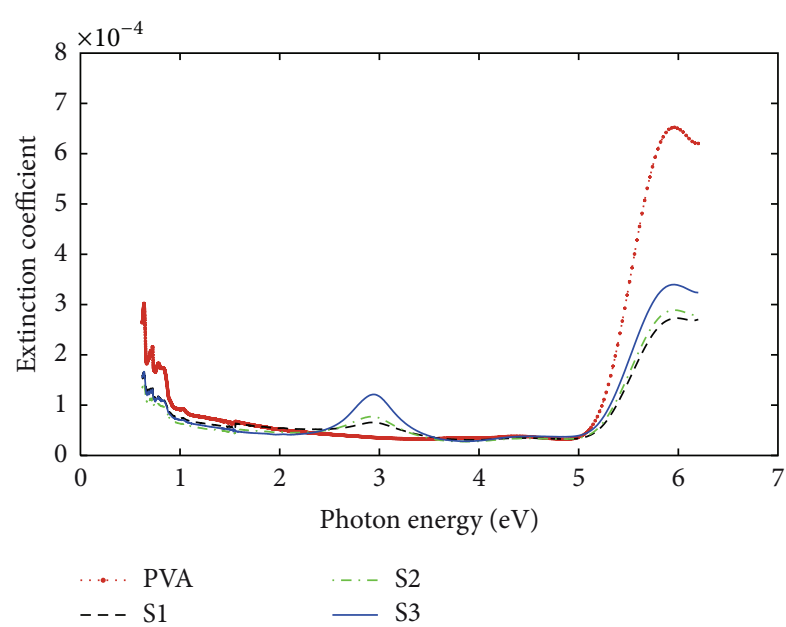

Figure 9: The extinction coefficient spectrum of films.

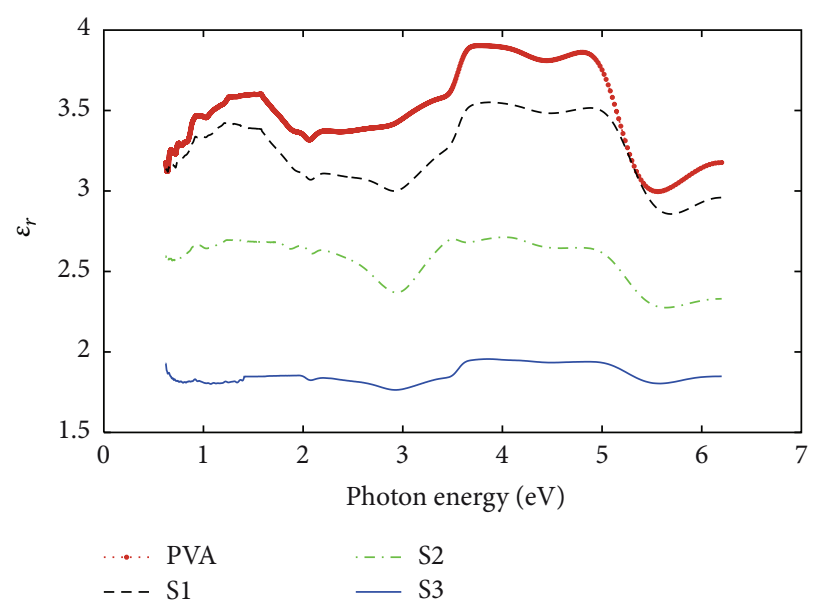

Figure 10: Real part of the dielectric constant of pure PVA and Ag nanoparticle doped films.

The real part of dielectric constant is related to the dispersion. In order to explain the dispersion it is necessary to take into account the actual motion of the electrons in the optical medium through which the light is traveling. The imaginary part represents the dissipative rate of electromagnetic wave propagation in the medium. The real and imaginary parts dependences on photon energy of samples are shown in Figures 10 and 11, respectively. It can be concluded that $\varepsilon_{r}$ is larger than $\varepsilon_{i}$ because it mainly depends on $n^{2}$. With increasing the amount of silver nanoparticles in PVA, the real part of dielectric constant is decreased. It is due to decrease of the dielectric property of films because of Ag nanoparticles metal lattice in the host PVA polymer matrix. The normal dispersion is associated with an increase in $\operatorname{Re}(\varepsilon)$ with $\omega$, and anomalous dispersion is associated with the reverse mechanism. Normal dispersion is occurred everywhere expect in the neighborhood of the resonance frequency. In this region the anomalous dispersion is appreciable. Since a positive imaginary part to $\varepsilon_{i}$ represents dissipation of energy from the electromagnetic wave into the medium, the regions where $\varepsilon_{i}$

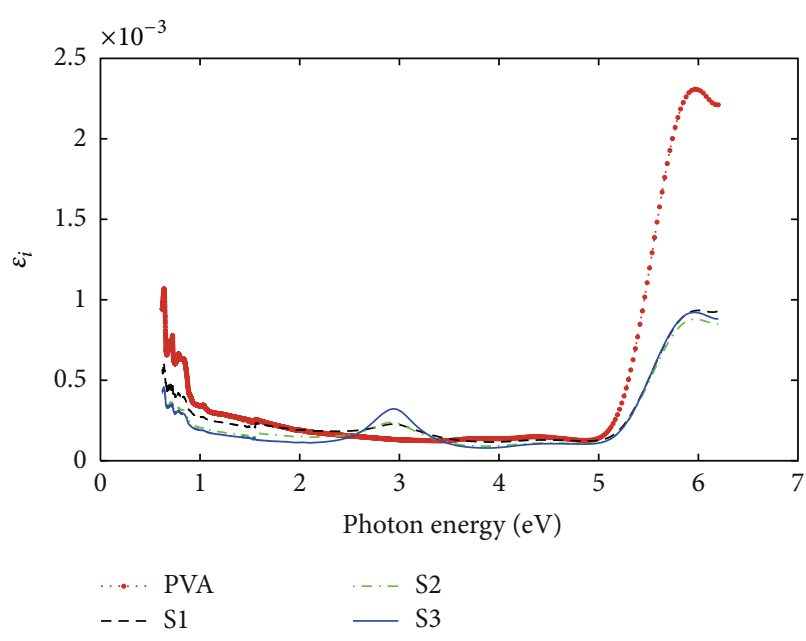

FIGURE 11: Imaginary part of the dielectric function of films.

is large are called regions of resonant absorption [47]. The peak that appears in Figure 11 at $E=2.95 \mathrm{eV}$ shows the plasmonic absorption.

The complex dielectric function and complex optical conductivity are introduced through Maxwell's equations. The interband transitions have threshold energy at the energy gap. That is, we expect the frequency dependence of the real part of the conductivity $\sigma_{r}(\omega)$ due to an interband transition to exhibit a threshold for an allowed electronic transition [48]. In interband transition, real $\sigma_{r}$ and imaginary $\sigma_{i}$ components of optical conductivity are described as [4850]

$$
\begin{aligned}
& \sigma_{r}=\omega \varepsilon_{i} \varepsilon_{0}, \\
& \sigma_{i}=\omega \varepsilon_{r} \varepsilon_{0},
\end{aligned}
$$

where $\omega$ is the angular frequency of electromagnetic wave and $\varepsilon_{0}$ is the free space dielectric constant. The real and imaginary parts of the optical conductivity dependence on the photon energy are shown in Figures 12 and 13, respectively.

The real optical conductivity in bandgap energy region after doping the Ag-nanoparticles in PVA polymer decreases. It can be due to the segregation effect $[41,51]$. The segregated effect is the dispersion of metallic particles restricted by the presence of much larger polymeric particles. The observed effect of Ag nanoparticles on the optical conductivity and conduction behavior of PVA films can be explained on the basis of charge transfer complex formation involving PVA molecules and the dopant. When PVA polymer is mixed with Ag nanoparticles, as a result, the filler is pushed into interstitial space between the polymer particles and forms a segregated network [51]. At higher dopant concentration, there may be segregation of the dopant in the polymer matrix which decreases the conductivity. Thus motion of charge carriers or localized fluctuations of charged particles in molecular aggregates impedes optical conductivity. Moreover, there is a weak peak at $E=2.95 \mathrm{eV}$ that can be seen only in the doped samples. This new peak is attributed to the formation of charge transfer or the surface plasmon 


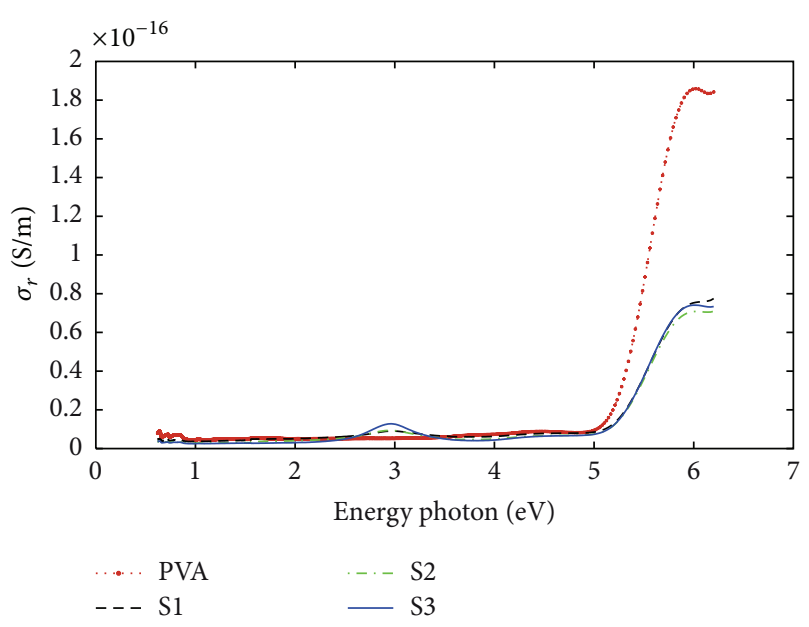

FIGURE 12: Real part of the optical conductivity of pure PVA and doped films.

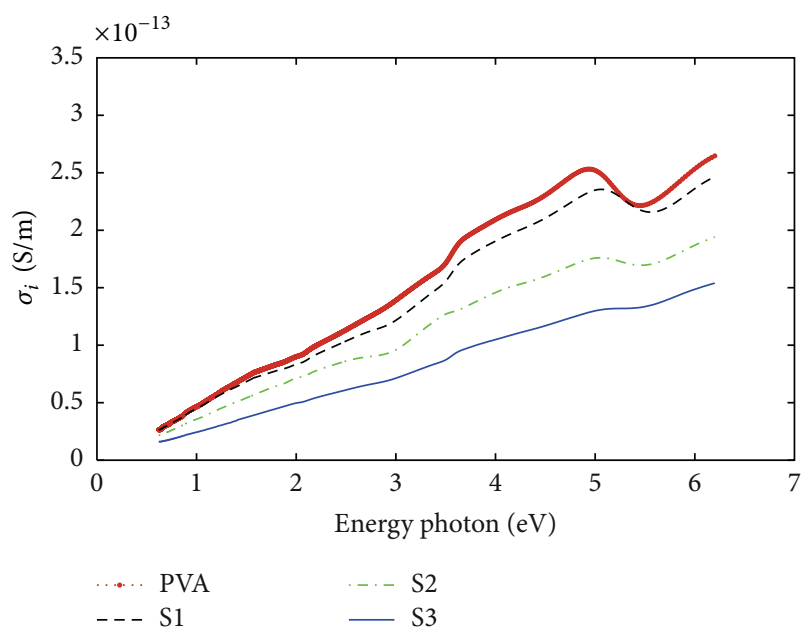

FIGURE 13: Imaginary part of the optical conductivity of pure PVA and doped films.

silver nanoparticles. Another result is that by increasing the concentration of Ag nanoparticles in PVA matrix, the imaginary part of optical conductivity decreases.

A dielectric sample in an external electric field acquires a nonzero macroscopic dipole moment indicating that the dielectric is polarized under the influence of the field. Polarization of dielectric material achieves its equilibrium value, not instantaneously but rather over a period of time $\tau$ [52]. The dielectric relaxation time $\tau$ can be evaluated using

$$
\tau=\frac{\varepsilon_{\infty}-\varepsilon_{r}}{\omega \varepsilon_{i}}
$$

Figure 14 shows the dielectric relaxation time as a function of photon energy for pure PVA polymer and Ag nanoparticle doped PVA films. The valley at $2.95 \mathrm{eV}$ for doped samples increases with increasing the concentration of Ag nanoparticles in the structure of the films. In other words the relaxation time of dipole orientation decreases.

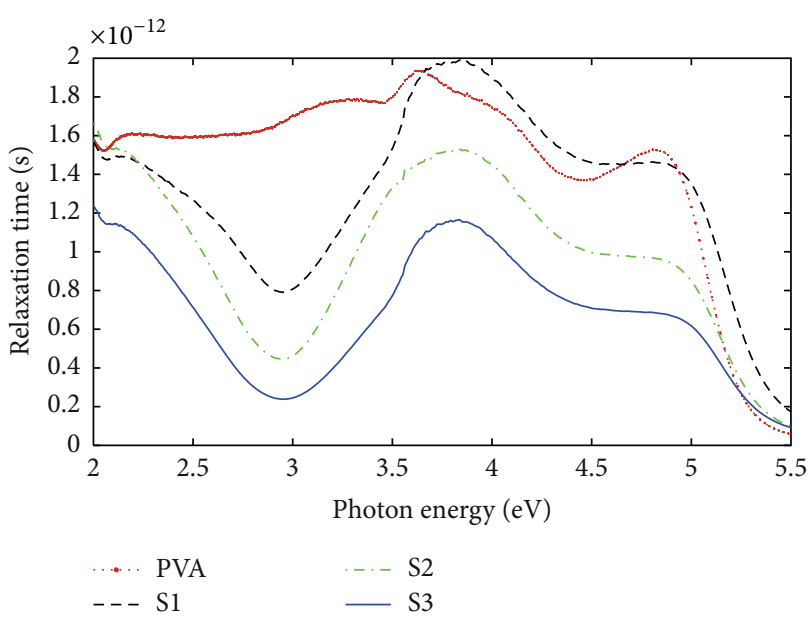

FIGURE 14: Relaxation time versus photon energy of films.

This is another reason for decreasing the dielectric function and increasing the conductivity of films with increasing the concentration of nanoparticles in their structure [53].

Refractive index dispersion is a determinant factor in optical materials. It is a significant factor in optical communication and in designing devices for spectral dispersion [35]. In the normal dispersion region, the refractive index dispersion has been analyzed using the single oscillator model developed by theory of Wemple and DiDomenico [54]. They introduced a dispersion-energy parameter $E_{d}$, which connects the coordination number and the charge distribution within each unit cell. $E_{d}$ is closely related to chemical bonding. Also they defined a single oscillator parameter $E_{0}$, which is proportional to the energy of oscillator. In terms of this dispersion energy $E_{d}$ and single oscillator energy $E_{0}$, the refractive index $n$ at frequency can be written as

$$
\frac{1}{n^{2}-1}=\frac{E_{0}}{E_{d}}-\frac{1}{E_{0} E_{d}}(h v)^{2}
$$

The values of $E_{d}$ and $E_{0}$ can be obtained from the intercept and slope of the linear part of $\left(n^{2}-1\right)^{-1}$ plot versus $(h \nu)^{2}$ as is shown in Figure 15. Also, dispersion of refractive index is controlled by the combined effects of $E_{d}$ and $E_{0}$. The calculated values of the dispersion parameters $\left(E_{0}\right.$ and $\left.E_{d}\right)$ as well as the corresponding optical constant $\left(\varepsilon=n^{2}\right)$ for the pure PVA film and samples 1 to 3 are listed in Table 1 . The dispersion energy values decreases with increasing the concentration of Ag nanoparticles in PVA films, because the anion strength of the dielectric medium has been declined. Therefore, the PVA polymer host is less willing to keep the electrons in their outer layers. The single oscillator energy is an average energy gap as pointed out in many references [50]. The $E_{0}$ value of the films is related empirically to the lowest indirect bandgap by $E_{0} \approx 1.03 E_{g}$. This relation is in good agreement with the single oscillator model. 


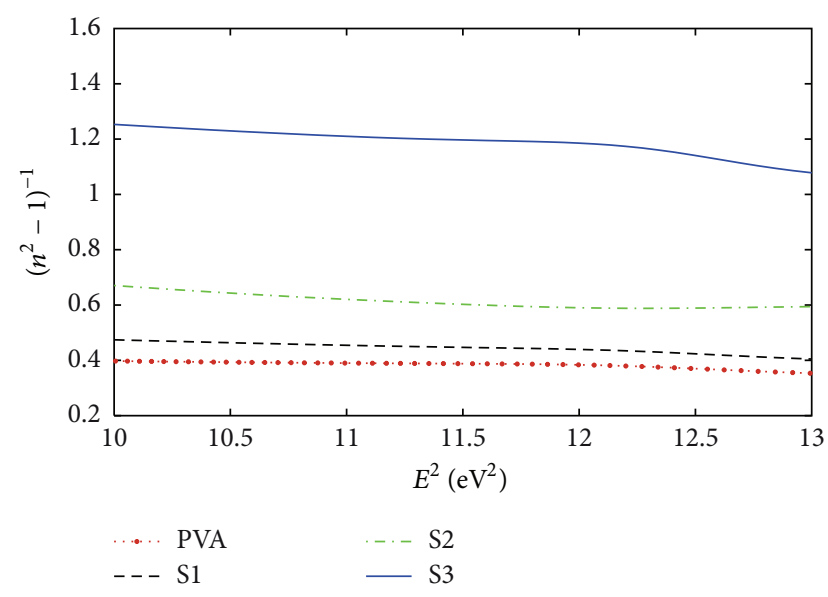

Figure 15: Plot of $\left(n^{2}-1\right)$ versus squared photon energy of pure PVA and doping samples.

TABLE 1: Dispersion parameters of films.

\begin{tabular}{lcccc}
\hline Samples & $n$ & $\varepsilon$ & $E_{0}$ & $E_{d}$ \\
\hline PVA & 1.56 & 2.44 & 5.19 & 7.49 \\
S1 & 1.48 & 2.19 & 5.04 & 6.03 \\
S2 & 1.37 & 1.89 & 4.97 & 4.46 \\
S3 & 1.19 & 1.42 & 4.87 & 2.05 \\
\hline
\end{tabular}

\section{Conclusions}

Preparation of silver nanoparticles by laser ablation method at different fluencies of laser pulse in pure water is investigated. The TEM analysis revealed that generated nanoparticles in this experimental condition are almost spherical and their average size was $6-12 \mathrm{~nm}$ and with increasing the laser pulse fluence, the size of nanoparticles is decreased. The Xray diffraction spectrum reveals that the number of crystallographic planes at certain angles is increased after doping Ag nanoparticles in the structure of PVA. FTIR spectrum peaks correspond to molecular vibrations and chemical bonds, indicate the presence of silver in the PVA polymer structure. The optical bandgap energy of the samples is decreased with increasing the concentrations of silver nanoparticles. Refractive index and dielectric constant are decreased with increasing the concentration of Ag nanoparticles. Increase of dopant concentration resulted in a decrease in real part of the optical conductivity because of segregation effect. The refractive index and consequently the related dispersion parameters of PVA and doped PVA have been determined and explained using the Wemple-DiDomenico model.

\section{References}

[1] A. Nimrodh Ananth and S. Umapathy, "On the optical and thermal properties of in situ/ex situ reduced Ag NP's/PVA composites and its role as a simple SPR-based protein sensor," Applied Nanoscience, vol. 1, no. 2, pp. 87-96, 2011.

[2] G. Nesher, G. Marom, and D. Avnir, "Metal-polymer composites: synthesis and characterization of polyaniline and other polymer at Silver compositions," Chemistry of Materials, vol. 20, no. 13, pp. 4425-4432, 2008.

[3] P.-H. Wang, Y.-Z. Wu, and Q.-R. Zhu, "Polymermetal composite particles: polymer core and metal shell," Journal of Materials Science Letters, vol. 21, no. 23, pp. 1825-1828, 2002.

[4] S. Clémenson, P. Alcouffe, L. David, and E. Espuche, "Structure and morphology of membranes prepared from polyvinyl alcohol and silver nitrate: influence of the annealing treatment and of the film thickness," Desalination, vol. 200, no. 1-3, pp. 437439, 2006.

[5] K. Akamatsu, S. Takei, M. Mizuhata et al., "Preparation and characterization of polymer thin films containing silver and silver sulfide nanoparticles," Thin Solid Films, vol. 359, no. 1, pp. 55-60, 2000.

[6] R. Zeng, M. Z. Rong, M. Q. Zhang, H. C. Liang, and H. M. Zeng, "Laser ablation of polymer-based silver nanocomposites," Applied Surface Science, vol. 187, no. 3-4, pp. 239-247, 2002.

[7] S. Mahendia, A. K. Tomar, and S. Kumar, "Electrical conductivity and dielectric spectroscopic studies of PVA-Ag nanocomposite films," Journal of Alloys and Compounds, vol. 508, no. 2, pp. 406-411, 2010.

[8] N. Singh and P. K. Khanna, "In situ synthesis of silver nanoparticles in polymethylmethacrylate," Materials Chemistry and Physics, vol. 104, no. 2-3, pp. 367-372, 2007.

[9] P. K. Khanna, R. Gokhale, V. V. V. S. Subbarao, A. K. Vishwanath, B. K. Das, and C. V. V. Satyanarayana, "PVA stabilized gold nanoparticles by use of unexplored albeit conventional reducing agent," Materials Chemistry and Physics, vol. 92, no. 1, pp. 229-233, 2005.

[10] K. Akamatsu, S. Takei, M. Mizuhata et al., "Preparation and characterization of polymer thin films containing silver and silver sulfide nanoparticles," Thin Solid Films, vol. 359, no. 1, pp. 55-60, 2000.

[11] I. Hussain, M. Brust, A. J. Papworth, and A. I. Cooper, "Preparation of acrylate-stabilized gold and silver hydrosols and gold-polymer composite films," Langmuir, vol. 19, no. 11, pp. 4831-4835, 2003.

[12] S. A. Zavyalov, A. N. Pivkina, and J. Schoonman, "Formation and characterization of metal-polymer nanostructured composites," Solid State Ionics, vol. 147, no. 3-4, pp. 415-419, 2002.

[13] J. Lee, D. Bhattacharyya, A. J. Easteal, and J. B. Metson, "Properties of nano- $\mathrm{ZnO} /$ poly(vinyl alcohol)/poly(ethylene oxide) composite thin films," Current Applied Physics, vol. 8, no. 1, pp. $42-47,2008$.

[14] Z. Zhong, D. Wang, Y. Cui, M. W. Bockrath, and C. H. Lieber, "Nanowire crossbar arrays as address decoders for integrated nanosystems," Science, vol. 302, no. 5649, pp. 1377-1379, 2003.

[15] D. S. Hopkins, D. Pekker, P. M. Goldbart, and A. Bezryadin, "Quantum interference device made by DNA templating of superconducting nanowires," Science, vol. 308, no. 5729, pp. 1762-1765, 2005.

[16] S. Clémenson, D. Léonard, D. Sage, L. David, and E. Espuche, "Metal nanocomposite films prepared in Situ from PVA and silver nitrate. Study of the nanostructuration process and morphology as a function of the in Situ routes," Journal of Polymer Science A, vol. 46, no. 6, pp. 2062-2071, 2008.

[17] M. K. Temgire and S. S. Joshi, "Optical and structural studies of silver nanoparticles," Radiation Physics and Chemistry, vol. 71, no. 5, pp. 1039-1044, 2004.

[18] M. Zheng, M. Gu, Y. Jin, and G. Jin, "Optical properties of silverdispersed PVP thin film," Materials Research Bulletin, vol. 36, no. 5-6, pp. 853-859, 2001. 
[19] O. L. A. Monti, J. T. Fourkas, and D. J. Nesbitt, "Diffractionlimited photogeneration and characterization of silver nanoparticles," Journal of Physical Chemistry B, vol. 108, no. 5, pp. 16041612, 2004.

[20] K. L. Kelly, E. Coronado, L. L. Zhao, and G. C. Schatz, "The optical properties of metal nanoparticles: the influence of size, shape, and dielectric environment," Journal of Physical Chemistry B, vol. 107, no. 3, pp. 668-677, 2003.

[21] H. Weickmann, J. C. Tiller, R. Thomann, and R. Mülhaupt, "Metallized organoclays as new intermediates for aqueous nanohybrid dispersions, nanohybrid catalysts and antimicrobial polymer hybrid nanocomposites," Macromolecular Materials and Engineering, vol. 290, no. 9, pp. 875-883, 2005.

[22] U. Keirbeg and M. Vollmer, Optical Properties of Metal Clusters, vol. 25 of Springer Series in Material Science, 1995.

[23] S. Chen and J. M. Sommers, "Alkanethiolate-protected copper nanoparticles: spectroscopy, electrochemistry, and solid-state morphological evolution," Journal of Physical Chemistry B, vol. 105, no. 37, pp. 8816-8820, 2001.

[24] A. A. Scalisi, G. Compagnini, L. D’Urso, and O. Puglisi, "Nonlinear optical activity in $\mathrm{Ag}-\mathrm{SiO}_{2}$ nanocomposite thin films with different silver concentration," Applied Surface Science, vol. 226, no. 1-3, pp. 237-241, 2004.

[25] G. Yang, W. Wang, Y. Zhou, H. Lu, G. Yang, and Z. Chen, "Linear and nonlinear optical properties of $\mathrm{Ag}$ nanocluster $/ \mathrm{BaTiO}_{3}$ composite films," Applied Physics Letters, vol. 81, no. 21, pp. 3969-3971, 2002.

[26] H. B. Liao, R. F. Xiao, H. Wang, K. S. Wong, and G. K. L. Wong, "Large third-order optical nonlinearity in $\mathrm{Au}: \mathrm{TiO}_{2}$ composite films measured on a femtosecond time scale," Applied Physics Letters, vol. 72, no. 15, pp. 1817-1819, 1998.

[27] A. K. Sarychev, D. J. Bergman, and Y. Yagil, "Theory of the optical and microwave properties of metal-dielectric films," Physical Review B, vol. 51, no. 8, pp. 5366-5385, 1995.

[28] G. Mie, "Beiträge zur Optik trüber Medien, speziell kolloidaler Metallösungen," Annalen der Physik, vol. 330, pp. 377-445, 1908.

[29] R. Gans, "Über die Form ultramikroskopischer Silberteilchen," Annalen der Physik, vol. 352, no. 10, pp. 270-284, 1915.

[30] S. Link and M. A. El-Sayed, "Spectral properties and relaxation dynamics of surface plasmon electronic oscillations in gold and silver nanodots and nanorods," Journal of Physical Chemistry B, vol. 103, no. 40, pp. 8410-8426, 1999.

[31] G. Fussell, J. Thomas, J. Scanlon, A. Lowman, and M. Marcolongo, "The effect of protein-free versus protein-containing medium on the mechanical properties and uptake of ions of PVA/PVP hydrogels," Journal of Biomaterials Science, vol. 16, no. 4, pp. 489-503, 2005.

[32] T. Tsuji, T. Mizuki, S. Ozono, and M. Tsuji, "Laser-induced silver nanocrystal formation in polyvinylpyrrolidone solutions," Journal of Photochemistry and Photobiology A, vol. 206, no. 2-3, pp. 134-139, 2009.

[33] J.-P. Sylvestre, S. Poulin, A. V. Kabashin, E. Sacher, M. Meunier, and J. H. T. Luong, "Surface chemistry of gold nanoparticles produced by laser ablation in aqueous media," Journal of Physical Chemistry B, vol. 108, no. 43, pp. 16864-16869, 2004.

[34] A. Gautam and S. Ram, "Preparation and thermomechanical properties of Ag-PVA nanocomposite films," Materials Chemistry and Physics, vol. 119, no. 1-2, pp. 266-271, 2010.

[35] I. Saini, J. Rozra, N. Chandak, S. Aggarwal, P. K. Sharma, and A. Sharma, "Tailoring of electrical, optical and structural properties of PVA by addition of Ag nanoparticles," Mater. Chem. Phys, vol. 139, pp. 802-810, 2013.
[36] S. Mahendia, A. K. Tomar, and S. Kumar, "Nano-Ag doping induced changes in optical and electrical behaviour of PVA films," Materials Science and Engineering B, vol. 176, no. 7, pp. 530-534, 2011.

[37] M. Abdelaziz and E. M. Abdelrazek, "Effect of dopant mixture on structural, optical and electron spin resonance properties of polyvinyl alcohol," Physica B, vol. 390, no. 1-2, pp. 1-9, 2007.

[38] B. Suo, X. Su, J. Wu, D. Chen, A. Wang, and Z. Guo, "Poly (vinyl alcohol) thin film filled with CdSe-ZnS quantum dots: fabrication, characterization and optical properties," Materials Chemistry and Physics, vol. 119, no. 1-2, pp. 237-242, 2010.

[39] B. Karthikeyan, "Spectroscopic studies on Ag-polyvinyl alcohol nanocomposite films," Physica B, vol. 364, no. 1-4, pp. 328-332, 2005.

[40] A. S. Kutsenko and V. M. Granchak, "Photochemical synthesis of silver nanoparticles in polyvinyl alcohol matrices," Theoretical and Experimental Chemistry, vol. 45, no. 5, pp. 313-318, 2009.

[41] C. U. Devi, A. K. Sharma, and V. V. R. N. Rao, "Electrical and optical properties of pure and silver nitrate-doped polyvinyl alcohol films," Materials Letters, vol. 56, no. 3, pp. 167-174, 2002.

[42] B. G. Streetman, Solid State Electronic Devices, Prentice Hall, New Jersey, NJ, USA, 3rd edition, 1990.

[43] J. Tauc and R. Grigorovici, "Optical properties and electronic structure of amorphous germanium," Physica Status Solidi (B), vol. 15, no. 2, pp. 627-637, 1966.

[44] J. Rozra, I. Saini, A. Sharma et al., "Cu nanoparticles induced structural, optical and electrical modification in PVA," Materials Chemistry and Physics, vol. 134, no. 2-3, pp. 1121-1126, 2012.

[45] M. Abdelaziz, "Cerium (III) doping effects on optical and thermal properties of PVA films," Physica B, vol. 406, no. 6-7, pp. 1300-1307, 2011.

[46] C. Kittle, Introduction to Solid State Physics, vol. 405, John Wiley \& Sons, New York, NY, USA, 1971.

[47] J. D. Jackson, Classical Electrodynamics, John Wiley \& Sons, 3rd edition, 1999.

[48] M. S. Dresselhaus, Solid State Physics Part II Optical Properties of Solids, vol. 6, 2001.

[49] J. N. Hodgson, Optical Absorption and Dispersion in Solids, Chapman \& Hall, London, UK, 1971.

[50] Y. Caglar, S. Ilican, and M. Caglar, "Single-oscillator model and determination of optical constants of spray pyrolyzed amorphous $\mathrm{SnO}_{2}$ thin films," European Physical Journal B, vol. 58, no. 3, pp. 251-256, 2007.

[51] Y. Seok Kim, Electrical Conductivity of Segregated Network Polymer Nanoposites, vol. 137, 2007.

[52] Y. Feldman, A. Puzenko, and Y. Ryabov, "Dielectric relaxation phenomena in complex materials," in Advances in Chemical Physics, vol. 133, pp. 1-125, Department of Applied Physics, The Hebrew University of Jerusalem, Jerusalem, Israel, 2006.

[53] S. Mahendia, A. K. Tomar, R. P. Chahal, P. Goyal, and S. Kumar, "Optical and structural properties of poly(vinyl alcohol) films embedded with citrate-stabilized gold nanoparticles," Journal of Physics D, vol. 44, no. 20, Article ID 205105, 2011.

[54] S. H. Wemple and M. DiDomenico, "Behavior of the electronic dielectric constant in covalent and ionic materials," Physical Review B, vol. 3, no. 4, pp. 1338-1351, 1971. 

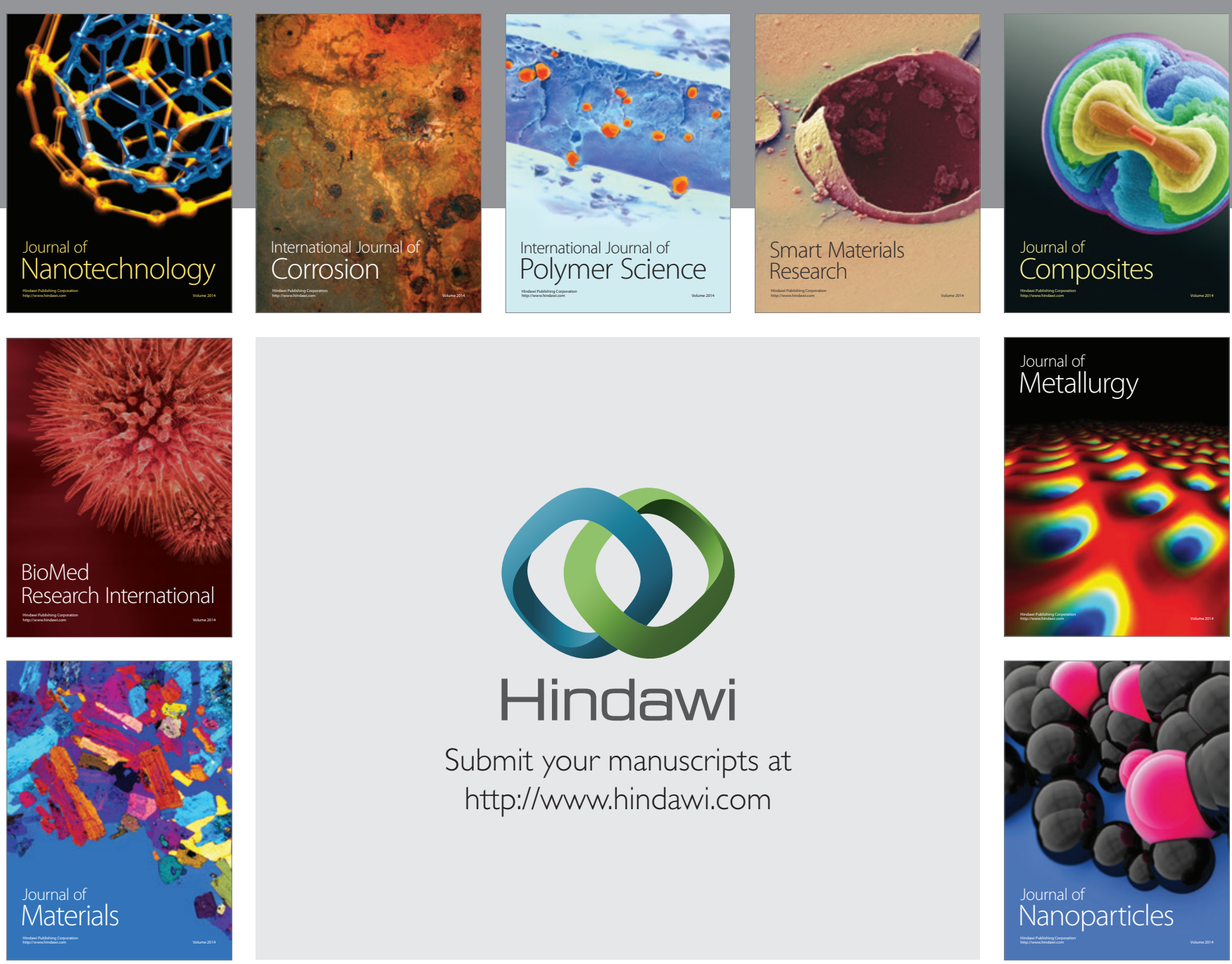

Submit your manuscripts at http://www.hindawi.com
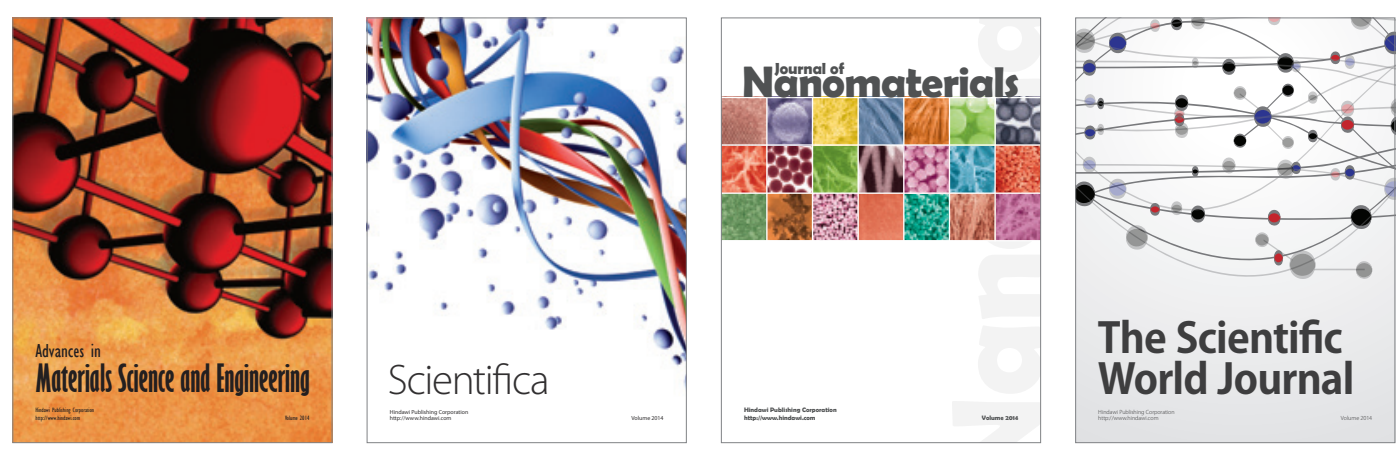

\section{The Scientific World Journal}
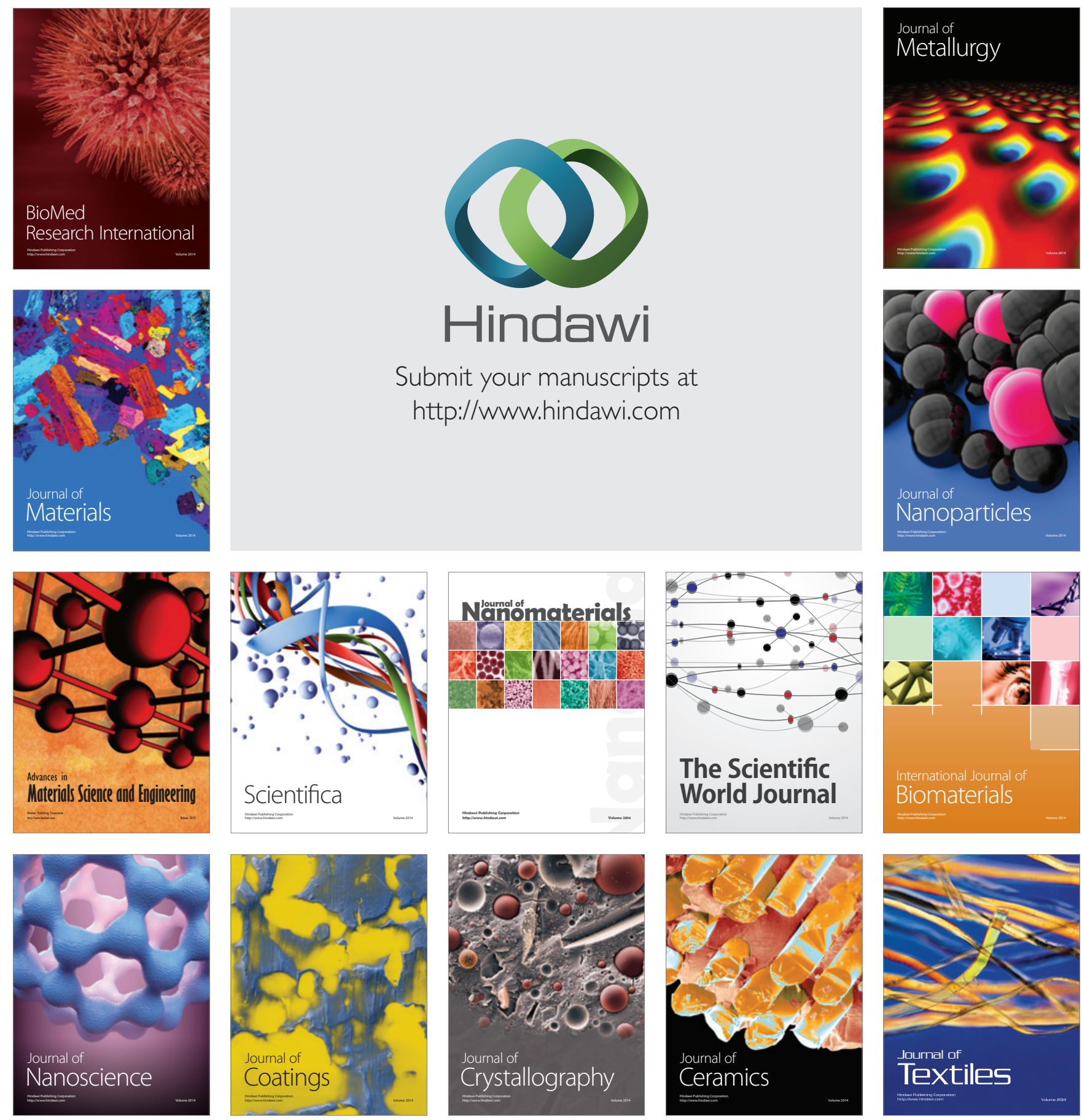